\title{
CUSTO DE PRODUÇÃo DAS BIOMASSAS DE EUCALIPTO E CAPIM-ELEFANTE PARA ENERGIA
}

\author{
Laurent Marie Roger Quéno ${ }^{1}$, Álvaro Nogueira de Souza², Humberto Ângelo ${ }^{3}$, \\ Ailton Teixeira do Vale ${ }^{4}$ Ildeu Soares Martins ${ }^{5}$
}

(recebido: 9 de fevereiro de 2010; aceito: 27 de maio de 2011)

\begin{abstract}
RESUMO: Nesse estudo, objetivou-se estabelecer o custo de produção da unidade energética gerada pela biomassa do eucalipto (Eucalyptus sp.), em diferentes tratamentos silviculturais, e pela biomassa do capim-elefante (Pennisetum sp.), e aplicar uma análise de sensibilidade para verificar as influências de fatores como o tratamento silvicultural do eucalipto, a produção volumétrica de cada espécie, o custo da terra e a taxa de juros nos custos de produção analisados. Foi demonstrado que o tratamento do eucalipto em plantio adensado e em curtíssima rotação de dois anos, com reforma do povoamento a cada seis anos, teve um custo médio de produção (CMPr) superior ao sistema convencional com rotação a cada seis anos e reforma aos 18. Observou-se, também, que o eucalipto apresenta, em média, um CMPr de $\mathrm{R} \$ 4,41 / \mathrm{Gj}$, inferior ao do capim-elefante, que, em média, é de $\mathrm{R} \$ 5,44 / \mathrm{Gj}$, mesmo com a capacidade que o último tem de produção anual superior de matéria seca. O capim-elefante tem a possibilidade de competir com o eucalipto quando um conjunto de condições se forma como taxa de juros igual ou superior a $8 \%$ a.a., preço da terra elevado e volume do capim-elefante igual ou acima de 35 toneladas de matéria seca por hectare por ano.
\end{abstract}

Palavras-chave: Eucalyptus sp., Pennisetum sp., biomassa, bioenergia, custo médio de produção.

\section{PRODUCTION COST OF BIOMASSES FROM EUCALYPTUS AND ELEFANT GRASS FOR ENERGY}

\begin{abstract}
This work established the unit energy cost generated from biomass of eucalyptus (Eucalyptus sp.) and elephant grass (Pennisetum sp.) and applied a sensitivity analysis to verify the influences of factors such as the silviculture of eucalyptus, production volume of each species, the cost of land and the interest rate. It was shown that the treatment of eucalyptus in very short rotation of 2 years with reform of stand every 6 years has a average cost of production higher than the traditional treatment of short rotation of 6 years with reform only at the age of 18. It was also observed that eucalyptus has a Production Cost on average of $R \$ 4,41 / G j$, lower than the elephant grass which is on average of $R \$ 5,44 / G j$, which however has a higher annual capacity of dry matter production. The elephant grass has the possibility to compete with eucalyptus when a set of conditions is met: discount rate higher than or equal to $8 \%$, High price of land, and elephant grass high volume production, greater than or equal to 35 tonnes of dry matter per hectare and year.
\end{abstract}

Key words: Eucalyptus sp., Pennisetum sp., biomass, bioenergy, average production cost.

\section{INTRODUÇÃO}

A procura de fontes alternativas para as energias fósseis tornou-se uma questão crucial para o futuro do desenvolvimento econômico do mundo. Entre as soluções possíveis, a biomassa se destaca como oportunidade a médio e longo prazo, especialmente no Brasil, que dispõe de terra em quantidade suficiente para alcançar uma produção significativa (GOLDEMBERG, 2009).
A biomassa vem adquirindo uma importância estratégica para o suprimento de energia em vários países, especialmente da União Européia. Portanto, a estimação do seu custo de produção é crucial para saber até qual nível pode ser uma fonte confiável, segura e accessível de energia (SMEETS; FAJJ, 2009).

Dois tipos de produção de biomassa lignocelulósica são desenvolvidos ao redor do mundo: as talhadias de curta rotação (TCR) ou short-rotation woody crops (SRWC), e

\footnotetext{
${ }^{1}$ Engenheiro Florestal, Doutorando em Ciências Florestais - Universidade de Brasília/UnB - Rua N37Qd40Lt20 - Anápolis city 2 etapa - 75094-420 Anápolis, GO - laurentfloresta@gmail.com

${ }^{2}$ Engenheiro florestal, Professor Dr. em Engenharia Florestal - Departamento de Engenharia Floresta1/EFL - Faculdade de Tecnologia/FT - Universidade de Brasília/UnB - Cx. P. 04357 - 70919-970 - Brasília, DF - ansouza@unb.br

${ }^{3}$ Engenheiro florestal, Professor Dr. em Economia e Política Florestal - Departamento de Engenharia Florestal/EFL - Faculdade de Tecnologia/FT Universidade de Brasília/UnB - Cx. P. 04357 - 70919-970 - Brasília, DF - humb@unb.br

${ }^{4}$ Engenheiro florestal, Professor Dr. em Energia na Agricultura - Departamento de Engenharia Florestal/EFL - Faculdade de Tecnologia/FT Universidade de Brasília/UnB - Cx. P. 04357 - 70919-970 - Brasília, DF - ailton.vale@gmail.com

${ }^{5}$ Engenheiro florestal, Professor Dr. em Genética e Melhoramento - Departamento de Engenharia Florestal/EFL - Faculdade de Tecnologia/FT Universidade de Brasília/UnB - Cx. P. 04357 - 70919-970 - Brasília, DF - ildmarti@unb.br
}

Cerne, Lavras, v. 17, n. 3, p. 417-426, jul./set. 2011 
as culturas energéticas herbáceas perenes ou herbaceous energy crops (HEC) (SENELWA et al., 1999).

Geralmente, nos países de clima temperado, as talhadias de curta rotação usam espécies, híbridos e clones dos gêneros Populus, Salix, Alnus, Betula, Liquidambar, Platanus, Acacia, Paulownia e Eucalyptus. Os plantios são cortados em ciclos cujas durações variam de 3 a 15 anos. A produção de matéria seca pode atingir, anualmente, de 8 a 16 toneladas por hectare em clima temperado. Com esse nível de produção, uma área de 8.000 hectares é suficiente para abastecer uma usina com ciclo a vapor, fornecendo $20 \mathrm{MW}$ (COOMBS, 2002).

As espécies herbáceas perenes que apresentam o potencial para uma produção anual de grande quantidade de biomassa têm as seguintes características fisiológicas: alta eficiência no processo de conversão da energia solar pela fotossíntese, sistema radicular extenso e profundo, uso ecônomo da água no solo e necessidade limitada de nutrientes, graças à sua capacidade de retranslocação destes, nas raízes, no final do ciclo vegetativo anual. Essas herbáceas de alta produtividade são, geralmente, de ciclo fotossintético $\mathrm{C} 4$ que é aproximadamente $40 \%$ mais eficiente para a captação de carbono que o mecanismo C3 das gramíneas mais comuns de clima temperado (SAMSON et al., 2005). No mundo, as principais espécies utilizadas são Panicum virgatum (Switchgrass), Phalaris arundinacea (Reed canary Grass), Miscanthus giganteus (E-grass), e pennisetum purpureum (Elephant Grass) conhecido no Brasil como capim-elefante (SMEETS et al., 2009).

O capim-elefante (Pennisetum purpureum Schum.) é uma planta originaria da África subtropical que foi descoberto em 1905 pelo Coronel Napier. A cultura chegou ao Brasil nos anos de 1920 e se encontra hoje adaptada às cinco regiões do país. Essa gramínea representa uma fonte importante de alimento para a criação de gado, especialmente para a alimentação do rebanho produtor de leite.

O capim-elefante apresenta a capacidade de produzir de 15 até 45 toneladas de matéria seca por hectare por ano, com alto teor de fibras e lignina, indicando seu potencial para energia (LOPES, 2004; QUESADA et al., 2004). Em função da adubação nitrogenada duas escolhas possíveis se destacam no Brasil para a produção de biomassa a partir do capim-elefante:

- A primeira escolha visa ao uso de genótipos de capim-elefante selecionados por sua alta capacidade de captar o nitrogênio atmosférico, graça à fixação biológica de nitrogênio (FBN). Essa linha de pesquisa é hoje liderada pela EMBRAPA Agrobiologia de Seropédica $(\mathrm{RJ})$.

- A segunda escolha visa à produção maximizada com adubação balanceada e utilização de híbrido de capim-elefante melhorado pelo cruzamento com outra espécie do gênero Pennisetum. Um cruzamento promissor foi conseguido entre o capim-elefante comum (Pennisetum purpureum Schum) e o milheto (Pennisetum glaucum). Esse capim foi obtido por Hanna, em 1980, na estação experimental de Tifton, no estado americano da Geórgia. Em 1995, ele foi introduzido no Brasil, pelo engenheiro agrônomo Herbert Vilela. Esse capim híbrido continuou sendo submetido a melhoramento genético até a obtenção de uma variedade adaptada para a produção de biomassa, conhecida como capim híbrido Carajás (VILELA, 2009)

Neste estudo, objetivou-se: estabelecer o custo de produção da unidade energética gerada pela biomassa do eucalipto (Eucalyptus sp.), em diferentes tratamentos silviculturais, e pela biomassa do capim-elefante (Pennisetum sp.); e, aplicar uma análise de sensibilidade para verificar as influências de fatores como o tratamento silvicultural do eucalipto, a produção volumétrica de cada espécie, o custo da terra e a taxa de juros nos custos de produção analisados.

\section{MATERIAL E MÉTODOS}

\subsection{Determinação da produção energética do eucalipto}

Os dados de produção volumétrica e energética do eucalipto usados neste estudo foram coletados pela equipe da RENABIO (Rede Nacional de Biomassa para Energia), num experimento realizado numa área da empresa ArcelorMittal BioEnergia, antiga ACESITA Energética, implantada em Itamarandiba (MG), na região do Vale do Jequitinhonha.

Vários tratamentos possíveis, com eucalipto para a produção de biomassa energética, foram testados, utilizando um clone de um híbrido de Eucalyptus grandis $\mathrm{x}$ Eucalyptus camaldulensis. Um delineamento experimental em blocos ao acaso foi instalado, no esquema de parcelas subdividas, onde a parcela foi representada pelo espaçamento e a subparcela pela idade. Três blocos foram instalados com os diferentes espaçamentos estudados. $\mathrm{O}$ espaçamento de $3 \mathrm{~m}$ entre linhas foi mantido por cada tratamento, variando o espaçamento na linha $(3 \mathrm{~m} \times 0,5 \mathrm{~m}$, $3 \mathrm{~m} \times 0,75 \mathrm{~m}, \ldots, 3 \mathrm{~m} \times 3 \mathrm{~m})$.

Cerne, Lavras, v. 17, n. 3, p. 417-426, jul./set. 2011 


\subsubsection{Produção de biomassa do eucalipto}

A partir do sexto mês, foram realizadas coletas semestrais dos dados de campo, medindo-se o diâmetro à altura do peito (DAP) e a altura total de oito árvores, selecionadas ao acaso, em cada tratamento. Para determinar o volume sólido $\left(\mathrm{m}^{3}\right)$ e a densidade, foi abatida e cubada a árvore média em cada tratamento. O método de Smalian, com seções a cada $1,5 \mathrm{~m}$, foi utilizado para estimar o volume das árvores. Conhecendo o volume da árvore média, foi possível calcular o volume por hectare.

A "densidade básica" $(\mathrm{Db})$ expressa a relação entre o peso absolutamente seco e o volume saturado da madeira. Conhecendo-se a densidade básica da madeira do eucalipto e o volume de madeira produzido por hectare, é possível fazer uma estimativa da quantidade de matéria seca produzida por hectare.

Na Tabela 1, apresenta-se a produção de matéria seca total gerada para cada tratamento, em função da idade. A quantidade de biomassa seca foi muito parecida entre tratamentos ao final dos seis anos.

Tabela 1 - Biomassa seca com casca (t/ha) produzida em cada tratamento.

Table 1 - Dry Eucalyptus biomass with bark (t/ha) produced in each treatment.

\begin{tabular}{lcrrrr}
\hline \multirow{2}{*}{$\begin{array}{l}\text { Espaçamento } \\
(\mathrm{m} \times \mathrm{m})\end{array}$} & \multicolumn{5}{c}{ Produção biomassa seca com casca - (t/ha) } \\
\cline { 2 - 6 } & 2 & 3 & \multicolumn{5}{c}{4} & \multicolumn{1}{c}{5} & 6 \\
\cline { 2 - 6 } & 48,0 & 77,8 & 107,5 & 113,3 & 119,0 \\
$3,0 \times 0,50$ & 45,0 & 73,0 & 101,0 & 111,3 & 121,5 \\
$3,0 \times 0,75$ & 40,8 & 66,3 & 91,8 & 106,6 & 121,4 \\
$3,0 \times 1,00$ & 33,8 & 62,1 & 90,5 & 105,8 & 121,2 \\
$3,0 \times 1,50$ & 31,2 & 56,4 & 81,6 & 99,8 & 118,0 \\
$3,0 \times 2,00$ & 28,4 & 54,1 & 79,9 & 98,1 & 116,4 \\
$3,0 \times 2,50$ & 25,2 & 51,5 & 77,8 & 96,0 & 114,2 \\
$3,0 \times 3,00$ & & & & & \\
\hline
\end{tabular}

Fonte: RENABIO (2009).

\subsubsection{Potencial energético do eucalipto}

O poder calorífico expressa a capacidade de geração de energia de um combustível durante a sua combustão. Sua unidade de medida é quilocaloria (kcal) por quilo $(\mathrm{kg})$ ou calorias (cal) por grama (g) de combustível. O poder calorífico foi calculado com uma bomba calorimétrica. Neste estudo, o poder calorífico superior foi utilizado para ter uma unidade comparável com a do capim-elefante.
$\mathrm{Na}$ Tabela 2, relata-se o poder calorífico superior da madeira, na idade de corte de cada tratamento. Seu maior valor foi atingido pelo plantio no espaçamento $3 \mathrm{~m} \times 2 \mathrm{~m}$, cortado aos seis anos de idade. Os valores são levemente inferiores aos encontrados na literatura, que ficam acima de $4.600 \mathrm{kcal} / \mathrm{kg}$ (VALE et al., 2000). A última coluna transforma o poder calorífico em Gigajoule por tonelada, um kcal sendo igual a $4 \cdot 186 \cdot 10^{-6} \mathrm{Gj}$.

Tabela 2 - Poder calorífico superior da madeira de eucalipto em função da densidade de plantio.

Table 2 - Eucalyptus wood Higher Heating Value depending on planting density.

\begin{tabular}{lccc}
\hline $\begin{array}{l}\text { Espaçamento do } \\
\text { plantio }(\mathrm{m} \times \mathrm{m})\end{array}$ & $(\mathrm{kcal} / \mathrm{kg})$ & $(\mathrm{kcal} / \mathrm{t})$ & $(\mathrm{Gj} / \mathrm{t})$ \\
\hline $3,0 \times 0,5$ & $4.464,50$ & 4.464 .500 & 18,69 \\
$3,0 \times 0,75$ & $4.484,75$ & 4.484 .750 & 18,77 \\
$3,0 \times 1,0$ & $4.505,00$ & 4.505 .000 & 18,86 \\
$3,0 \times 1,5$ & $4.503,00$ & 4.503 .000 & 18,85 \\
$3,0 \times 2,0$ & $4.551,10$ & 4.551 .100 & 19,05 \\
$3,0 \times 2,5$ & $4.531,00$ & 4.531 .000 & 18,97 \\
$3,0 \times 3,0$ & $4.510,80$ & 4.510 .800 & 18,88 \\
\hline
\end{tabular}

Fonte: Adaptado de RENABIO (2009).

Neste estudo, foram analisados sete tratamentos diferentes com eucalipto. Os três primeiros correspondem ao manejo em plantio adensado ( $3 \mathrm{~m} \times 0,5 \mathrm{~m}$ até $3 \mathrm{~m} \times 1 \mathrm{~m})$ em curtíssima rotação de dois anos (ultra-short rotation). A cada dois anos, o povoamento é cortado. Aos seis anos, após três rotações, o plantio é reformado para iniciar novo ciclo de três cortes.

No total, em 18 anos, o manejo em curtíssima rotação produz três ciclos de três cortes cada um. Essas altas densidades produzem uma quantidade alta de biomassa, permitindo um corte antecipado, com o inconveniente de se colher uma madeira em grande parte juvenil de menor poder calorífico. Os quatro outros tratamentos correspondem ao manejo convencional para produção de biomassa. São três rotações de seis anos, com condução da brotação nos dois últimos cortes. No total, após 18 anos nesse sistema, têm-se três cortes a partir do mesmo plantio.

O potencial de produção energético da biomassa é o resultado da combinação da quantidade de matéria seca produzida e de seu poder calorífico, enquanto a quantidade de matéria seca é o resultado da multiplicação do volume colhido de madeira por sua densidade básica.

Cerne, Lavras, v. 17, n. 3, p. 417-426, jul./set. 2011 


$$
P_{r} E=V \times D_{b} \times P_{c s}
$$

em que: $\mathrm{P}_{\mathrm{r}} \mathrm{E}=$ Produção de energia $(\mathrm{kcal} / \mathrm{ha})$,

$\mathrm{V}=$ Volume $\left(\mathrm{m}^{3} / \mathrm{ha}\right)$,

$D b=$ densidade básica $\left(\mathrm{kg} / \mathrm{m}^{3}\right)$, e

Pcs $=$ poder calorífico superior $(\mathrm{kcal} / \mathrm{kg})$.

Na Tabela 3, apresenta-se a produção energética encontrada por tratamento do eucalipto. O potencial energético pode ser expresso em calorias, mas também em joule ou em Watt $/ \mathrm{h}$. Os resultados foram transformados em gigajoule (GJ), o gigajoule é uma unidade internacionalmente utilizada nos estudos sobre a produção de biomassa para a energia e seu custo (CRUZ; NOGUEIRA, 2004; HOOGWIJK et al., 2009).

\subsection{Determinação da produção energética do capim- elefante}

Para a realização desse estudo, os dados usados sobre o capim-elefante foram encontrados na literatura. A análise de sensibilidade foi efetuada testando diversas produções de matéria seca possíveis para a região do cerrado. Os limites superiores de produção foram de 40 toneladas para o capim hibrido Carajás e 35 toneladas para a variedade com FBN. Esses limites superiores foram colocados, considerando o regime de chuva médio a baixo $(1.000 \mathrm{~mm}$ anuais), que caracteriza a região do Vale do Jequitinhonha. Estimou-se que a produção de capim-elefante FBN que não se recebe adubação nitrogenada é inferior ao híbrido Carajás em 5 toneladas para condições equivalente de sítio.

Neste estudo, para o poder calorífico superior da matéria seca do capim-elefante considerou-se o valor de $4200 \mathrm{kcal} / \mathrm{kg}$. Os valores encontrados na literatura oscilam entre $4.100 \mathrm{kcal} / \mathrm{cal}$ (FLORES, 2009) e $4.298 \mathrm{kcal} / \mathrm{kg}$ (VILELA, 2009).

\subsection{Cálculo do custo de produção das biomassas}

\subsubsection{Método do custo médio de produção}

De acordo com Rezende e Oliveira (2008), o método do custo médio de produção (CMPr) é aplicado quando se deseja trabalhar com o custo médio mínimo. Esse método é usado internacionalmente para apreciar o custo de produção de culturas energéticas perenes (BULLARD, 2001).

O CMPr resulta da relação entre o custo total atualizado $(\mathrm{CTj})$ e a produção total equivalente $(\mathrm{QTj})$. Ele foi utilizado para comparar: (i) o CMPr do eucalipto com rotações de seis anos com duas rebrotadas, sendo uma para 12 e outra para 18 anos, (ii) o CMPr do eucalipto em plantio adensado, reformado aos 6 anos e manejado com duas rebrotas em curtíssima rotação de dois anos, e (iii) o CMPr do capim-elefante com dois cortes anuais (um corte só o primeiro ano de implantação da cultura) e reformado a cada seis anos.

Sua fórmula é:

$$
C M P r=\frac{\sum_{j=0}^{n} C T j}{\sum_{j=0}^{n} Q T j}=\frac{\sum_{j=0}^{n} C_{j}(1+i)^{-j}}{\sum_{j=0}^{n} Q_{j}(1+i)^{-j}}
$$

em que: $\mathrm{CMPr}=$ custo médio de produção em reais/Gj

$\mathrm{CTj}=$ custo total atualizado

$\mathrm{QTj}=$ produção total equivalente

$n=$ duração do investimento

$j=$ período de tempo em que os custos e as quantidades produzidas ocorrem, $\mathrm{e}$

$i=$ taxa de juros.

Tabela 3 - Produções em incremento médio anual dos diferentes tratamentos considerados do eucalipto.

\begin{tabular}{|c|c|c|c|c|}
\hline Densidade de plantio & Tratamento & $\begin{array}{c}\text { Produção em volume } \\
\left(\mathrm{m}^{3} / \text { ha.ano }\right)\end{array}$ & $\begin{array}{c}\text { Produção de biomassa } \\
\text { (t.cc/ha.ano) }\end{array}$ & $\begin{array}{c}\text { Produção de energia } \\
(\mathrm{Gj} / \text { ha.ano })\end{array}$ \\
\hline $3 \mathrm{~m} \times 0,5 \mathrm{~m}$ & \multirow{3}{*}{$\begin{array}{l}\text { Cortes a cada dois anos } \\
\text { com reforma do plantio a } \\
\text { cada seis anos. }\end{array}$} & 50,00 & 24,00 & 448,52 \\
\hline $3 \mathrm{~m} \times 0,75 \mathrm{~m}$ & & 45,00 & 22,50 & 422,40 \\
\hline $3 \mathrm{~m} \mathrm{x} 1,00 \mathrm{~m}$ & & 40,00 & 20,40 & 384,70 \\
\hline $3 \mathrm{~m} \times 1,50 \mathrm{~m}$ & \multirow{4}{*}{$\begin{array}{l}\text { Um ciclo com três cortes } \\
\text { de } 6 \text { anos com condução da } \\
\text { rebrota. }\end{array}$} & 38,75 & 20,20 & 380,76 \\
\hline $3 \mathrm{~m} \times 2 \mathrm{~m}$ & & 37,85 & 19,67 & 374,67 \\
\hline $3 \mathrm{~m} \times 2,50 \mathrm{~m}$ & & 35,90 & 19,40 & 367,96 \\
\hline $3 \mathrm{~m} \times 3 \mathrm{~m}$ & & 34,00 & 19,03 & 359,39 \\
\hline
\end{tabular}

Table 3 - Productions from the different treatments of eucalyptus expressed in Mean Annual Increment.

Fonte: Adaptado de RENABIO (2009).

Cerne, Lavras, v. 17, n. 3, p. 417-426, jul./set. 2011 
2.3.2 Custo médio de produção da energia da biomassa do eucalipto

Para cada tratamento, os gastos foram contabilizados (Tabela 4) e atualizados com a taxa de juros para dar um resultado de custo total por hectare. $\mathrm{O}$ custo de colheita adotado foi o custo único para qualquer tratamento de $\mathrm{R} \$ 8,75 / \mathrm{m}^{3} \mathrm{cc}$; esse custo compreende o corte com um feller-buncher, a remoção com um skidder e o corte de toras com o processador.

O custo da terra foi contabilizado como despesa no início do projeto e como receita ao final, tendo seu valor sido reajustado em função da taxa de juros e do tempo. O valor da terra que foi integrado ao cálculo corresponde ao seu valor líquido, ou seja, ao custo real para o empreendimento (SILVA et al., 2008).
2.3.3 Custo Médio de Produção da energia da biomassa do capim elefante

Foram considerados três ciclos de 6 anos, partindo na atualização do ano 0 até o ano 17. As duas situações foram estudadas: A primeira situação baseou-se numa limitação da fertilização nitrogenada, graça à fixação biológica de nitrogênio (FBN). Os custos contabilizados (Tabela 5) estão disponíveis em Mazarella e Urquiaga (2006).

Na segunda situação (Tabela 6), procurou-se a otimização da produção com adubação balanceada e utilização do capim híbrido Carajás. A metodologia seguida para estimar os custos foi baseada no documento do Instituto Tecnológico do Paraná - TECPAR, editado pelo Sistema Brasileiro de Reposta Técnica (LEITE, 2005). Os custos da adubação provêm do nível preconizado por Vilela (2009).

Tabela 4 - Custos contabilizados para manejo do eucalipto.

Table 4 - Registered costs for eucalyptus management.

\begin{tabular}{|c|c|c|c|c|c|c|c|c|c|}
\hline \multirow{2}{*}{ Ano } & \multicolumn{4}{|c|}{ Plantio adensado de eucalipto } & \multicolumn{5}{|c|}{ Plantio convencional de eucalipto } \\
\hline & Operações & $3 \mathrm{~m} \times 0,5 \mathrm{~m}$ & $3 \mathrm{~m} \times 0,75 \mathrm{~m}$ & $3 \mathrm{~m} \times 1 \mathrm{~m}$ & Operações & $3 \mathrm{~m} \times 1,5 \mathrm{~m}$ & $3 \mathrm{~m} \times 2 \mathrm{~m}$ & $3 \mathrm{~m} \times 2,5 \mathrm{~m}$ & $3 \mathrm{~m} \times 3 \mathrm{~m}$ \\
\hline 0 & Impl.+M. & 4337,8 & 3726,3 & 3420,3 & Impl. + M. & 3074,4 & 2889,2 & 2779,6 & 2701,6 \\
\hline 1 & M. & 461,6 & 461,6 & 461,6 & M. & 499,7 & 499,7 & 499,7 & 499,7 \\
\hline 2 & $1^{\text {a }}$ Colh./M. & 1535,1 & 1447,7 & 1360,2 & M. & 125,4 & 125,4 & 125,4 & 125,4 \\
\hline 3 & M. $+1^{\mathrm{a}}$ Cond. & 363,0 & 363,0 & 363,0 & M. & 125,4 & 125,4 & 125,4 & 125,4 \\
\hline 4 & $2^{\mathrm{a}}$ Colh. $+\mathrm{M}$ & 1535,1 & 1447,7 & 1360,2 & M. & 125,4 & 125,4 & 125,4 & 125,4 \\
\hline 5 & M. $+2^{\mathrm{a} C o n d}$ & 363,0 & 363,0 & 363,0 & M. & 125,4 & 125,4 & 125,4 & 125,4 \\
\hline 6 & $3^{\mathrm{a}} \mathrm{C} .+\mathrm{R} .+\mathrm{M}$ & 4957,1 & 4258,1 & 3864,5 & $1^{\mathrm{a}}$ Colh. $+\mathrm{M}$ & 3152,2 & 3104,9 & 3002,6 & 2902,8 \\
\hline 7 & M. & 461,6 & 461,6 & 461,6 & M. $+1^{\text {a }}$ Cond. & 461,6 & 461,6 & 461,6 & 461,6 \\
\hline 8 & $1^{\text {a }}$ Colh. + M. & 1535,1 & 1447,7 & 1360,2 & M. $+1^{\text {a }}$ Cond. & 125,4 & 125,4 & 125,4 & 125,4 \\
\hline 9 & M. $+1^{\text {a }}$ Cond. & 363,0 & 363,0 & 363,0 & M. $+1^{\text {a }}$ Cond. & 125,4 & 125,4 & 125,4 & 125,4 \\
\hline 10 & $2^{\mathrm{a}}$ Colh. $+\mathrm{M}$ & 1535,1 & 1447,7 & 1360,2 & M. $+1^{\text {a }}$ Cond. & 125,4 & 125,4 & 125,4 & 125,4 \\
\hline 11 & M. $+2^{\mathrm{a}}$ Cond. & 363,0 & 363,0 & 363,0 & M. $+1^{\mathrm{a}}$ Cond. & 125,4 & 125,4 & 125,4 & 125,4 \\
\hline 12 & $3^{\mathrm{a} C} .+\mathrm{R} .+\mathrm{M}$. & 5029,7 & 4330,7 & 3937,1 & $2^{\mathrm{a}}$ Colh. $+\mathrm{M}$ & 3168,4 & 3121,2 & 3018,8 & 2919,1 \\
\hline 13 & M. & 461,6 & 461,6 & 461,6 & M. $+2^{\mathrm{a}}$ Cond. & 499,7 & 499,7 & 499,7 & 499,7 \\
\hline 14 & $1^{\mathrm{a}}$ Colh. $+\mathrm{M}$ & 1535,1 & 1447,7 & 1360,2 & M. $+2^{\mathrm{a}}$ Cond. & 125,4 & 125,4 & 125,4 & 125,4 \\
\hline 15 & M. $+1{ }^{\mathrm{a}}$ Cond. & 363,0 & 363,0 & 363,0 & M. $+2^{\mathrm{a}}$ Cond. & 125,4 & 125,4 & 125,4 & 125,4 \\
\hline 16 & $2^{\mathrm{a}}$ Colh. $+\mathrm{M}$ & 1535,1 & 1447,7 & 1360,2 & M. $+2^{\mathrm{a}}$ Cond. & 125,4 & 125,4 & 125,4 & 125,4 \\
\hline 17 & M. $+2^{\mathrm{a}}$ Cond. & 363,0 & 363,0 & 363,0 & M. $+2^{\mathrm{a}}$ Cond. & 125,4 & 125,4 & 125,4 & 125,4 \\
\hline 18 & $3^{\text {a Colh. }}$ & 874,9 & 787,4 & 699,9 & $3^{\text {a }}$ Colheita & 2034,1 & 1986,9 & 1884,5 & 1784,8 \\
\hline
\end{tabular}

Fonte: Adaptado de RENABIO (2009).

Impl. = Implantação; $\quad$ R. = Reforma;

M. = Manutenção;
Cond. $=$ Condução da rebrota.

Colh. $=$ Colheita;

Cerne, Lavras, v. 17, n. 3, p. 417-426, jul./set. 2011 
Tabela 5 - Custos por hectare do capim-elefante FBN (zero N/ha) num ciclo de 6 anos.

Table 5 - Costs per hectare of elephant-grass FBN (zero N/ha) in a 6 years period.

\begin{tabular}{lcc}
\hline Item de custo & $\begin{array}{c}\text { Ano de } \\
\text { ocorrência }\end{array}$ & $\begin{array}{c}\text { Custos por } \\
\text { hectare }\end{array}$ \\
\hline Formação & Ano 1 & 1981,77 \\
Colheita (um só corte) & Ano 1 & 603,70 \\
\hline Total & Ano 1 & 2585,47 \\
\hline Manutenção & Anos 2 a 6 & 617,91 \\
Colheita (dois cortes) & Anos 2 a 6 & 1207,40 \\
\hline Total por ano & Anos 2 a 6 & 1825,31 \\
\hline
\end{tabular}

Fonte: Adaptado de Mazarella e Urquiaga (2006).

Tabela 6 - Custos por hectare do capim híbrido Carajás.

Table 6 - Costs per hectare of hybrid Carajás elephant-grass.

\begin{tabular}{|c|c|c|c|}
\hline \multirow{2}{*}{ Item de custo } & \multicolumn{3}{|c|}{ Custo por hectare } \\
\hline & Insumos & Mecanização & Mão-de-obra \\
\hline $\begin{array}{l}\text { Custo de Formação, } \\
\text { Ano } 1\end{array}$ & 976,6 & 627,50 & 591 \\
\hline $\begin{array}{l}\text { Colheita: Um só corte, } \\
\text { Ano } 1\end{array}$ & & 603,5 & \\
\hline Total Ano 1 & & $2.794,60$ & \\
\hline $\begin{array}{l}\text { Custos Manutenção, } \\
\text { Ano } 2\end{array}$ & 510 & 307,50 & 190 \\
\hline $\begin{array}{l}\text { Colheita: Dois cortes, } \\
\text { Ano } 2\end{array}$ & & $1.207,00$ & \\
\hline Total Ano 2 & & $2.214,50$ & \\
\hline $\begin{array}{l}\text { Custos Manutenção, } \\
\text { Anos } 3 \text { a } 6\end{array}$ & 580 & 307,50 & 120 \\
\hline $\begin{array}{l}\text { Colheita: Dois cortes, } \\
\text { Anos } 3 \text { a } 6\end{array}$ & & 1.207 & \\
\hline Total Anos 3 a 6 & & $2.214,50$ & \\
\hline
\end{tabular}

Fonte: Adaptado de Leite (2005) e Vilela (2009)

Os custos de colheita utilizados neste trabalho foram publicados por Mazarella e Urquiaga (2006). Para a comparação entre os dados das culturas do capimelefante e do eucalipto, incluiu-se o custo de estradas (abertura e manutenção). Não foram incluídos os custos de administração para nenhuma das biomassas.

O ciclo de vida útil do capim-elefante é, normalmente, de cinco a sete anos. Os cálculos de custos para o eucalipto preveem uma duração total de 18 anos (três ciclos com reforma ou um ciclo com três conduções da rebrota). Por isso, os cálculos de custo do capimelefante foram feitos nesse mesmo horizonte de 18 anos, incluindo três ciclos totais de seis anos com implantação e manutenção e com dois cortes anuais.

\subsection{Análise de sensibilidade e estatística}

Foi realizada uma análise de sensibilidade para comparar o Custo Médio de Produção do eucalipto em diferentes densidades de plantio e idades de corte com o do capim-elefante nas duas condições de produção: o genótipo melhorado FBN e o capim híbrido Carajás. Assim, o comportamento econômico de cada produção foi analisado, variando-se a produção, a taxa de juros e o preço da terra. A taxa de juros foi testada entre os limites de $4 \%$ a $10 \%$ a.a comuns em projetos florestais. A taxa de juros de $6,75 \%$ a.a foi testada por ser aquela praticada pelo governo no financiamento de plantios florestais. $\mathrm{O}$ custo da terra foi analisado, considerando variações entre os limites de preços da região em estudo.

Uma análise de variância foi aplicada para comparar as médias dos resultados de CMPr das produções de eucalipto entre si, de capim-elefante entre si, e de todas as produções consideradas. $\mathrm{O}$ teste $\mathrm{F}$ a $1 \%$ ou a $5 \%$ de probabilidade foi realizado para determinar se os tratamentos foram estaticamente diferentes entre si.

Em seguida, foi empregado o teste de NewmanKeuls que é um teste de comparação das médias por pares, realizado ao final da ANOVA. O resultado do teste é uma série de pares de grupos cujas médias são consideradas como, significativamente, diferentes, ao nível de probabilidade a escolhida (PIMENTEL-GOMES; GARCIA, 2002).

\section{RESULTADOS E DISCUSSÃO}

\subsection{Custo de produção da unidade energética da biomassa de eucalipto}

Na Tabela 7, demonstra-se um arranjo dos resultados da análise de sensibilidade do custo de produção da biomassa de eucalipto. Foram realçados em negritos os tratamentos que permitem o menor custo para cada situação de taxa de juros e de preço da terra.

Analisando os resultados, pode-se observar que os tratamentos adensados em curtíssima rotação (dois anos), que permitem uma quantidade elevada de produção em termo de volume, apresentam um custo maior de produção que os tratamentos tradicionais. De todos os tratamentos, foram os plantios com densidades $3 \mathrm{~m} \times 2 \mathrm{~m}$ ou $3 \mathrm{~m} \times 2,5 \mathrm{~m}$ que apresentaram o menor CMPr. 
Tabela 7 - Custo médio de produção $(\mathrm{CMPr})$ da unidade energética $(\mathrm{R} \$ / \mathrm{Gj})$ de biomassa de eucalipto em função do tratamento, da taxa de juros e do preço da terra.

Table 7 - The unit energy Average Production Cost $(R \$ / G j)$ of eucalyptus biomass depending on the treatment, the discount rate and the land price.

\begin{tabular}{|c|c|c|c|c|c|c|c|c|c|}
\hline \multirow{2}{*}{\multicolumn{2}{|c|}{$\begin{array}{c}\text { Produção Média na Idade } \\
\text { de Colheita } \\
\left(\mathrm{m}^{3} / \mathrm{ha} / \mathrm{ano}\right)\end{array}$}} & \multicolumn{3}{|c|}{$\begin{array}{c}\text { Tratamento Adensado em } \\
\text { Curtíssima Rotação de } 2 \text { anos }\end{array}$} & \multicolumn{4}{|c|}{ Tratamento Tradicional em Rotação de 6 anos } & \multirow{5}{*}{$\begin{array}{c}\text { Espaçamento } \\
\text { do plantio } \\
\leftarrow\end{array}$} \\
\hline & & 50 & 45 & 40 & 38,75 & 37,85 & 35,9 & 34 & \\
\hline \multirow{3}{*}{$\begin{array}{c}\text { Taxa de } \\
\text { juros } \\
(\% / \mathrm{a} . \mathrm{a})\end{array}$} & \multirow{3}{*}{$\begin{array}{l}\text { Preço líquido } \\
\text { da terra } \\
(\mathrm{R} \$ / \mathrm{ha})\end{array}$} & $3 \mathrm{~m}$ & $3 \mathrm{~m}$ & $3 \mathrm{~m}$ & $3 \mathrm{~m}$ & $3 \mathrm{~m}$ & $3 \mathrm{~m}$ & $3 \mathrm{~m}$ & \\
\hline & & $\mathrm{X}$ & $\mathrm{x}$ & $\mathrm{x}$ & $\mathrm{x}$ & $\mathrm{x}$ & $\mathrm{X}$ & $\mathrm{x}$ & \\
\hline & & $0,5 \mathrm{~m}$ & $0,75 \mathrm{~m}$ & $1,00 \mathrm{~m}$ & $1,50 \mathrm{~m}$ & $2 \mathrm{~m}$ & $2,5 \mathrm{~m}$ & $3 \mathrm{~m}$ & \\
\hline \multirow{5}{*}{$10 \%$} & 4.000 & 5,20 & 5,07 & 5,26 & 4,53 & 4,49 & 4,49 & 4,51 & \multirow{25}{*}{$\begin{array}{l}\text { Custo médio } \\
\text { produção } \\
(\mathrm{R} \$ / \mathrm{Gj})\end{array}$} \\
\hline & 4.286 & 5,28 & 5,16 & 5,36 & 4,65 & 4,61 & 4,61 & 4,63 & \\
\hline & 5.000 & 5,49 & 5,37 & 5,59 & 4,94 & 4,91 & 4,91 & 4,95 & \\
\hline & 6.000 & 5,77 & 5,68 & 5,93 & 5,36 & 5,33 & 5,34 & 5,38 & \\
\hline & 7.000 & 6,06 & 5,98 & 6,26 & 5,77 & 5,75 & 5,77 & 5,82 & \\
\hline \multirow{5}{*}{$8 \%$} & 4.000 & 4,83 & 4,70 & 4,87 & 3,93 & 3,90 & 3,89 & 3,91 & \\
\hline & 4.286 & 4,90 & 4,77 & 4,95 & 4,03 & 4,00 & 3,99 & 4,01 & \\
\hline & 5.000 & 5,08 & 4,96 & 5,15 & 4,28 & 4,25 & 4,25 & 4,27 & \\
\hline & 6.000 & 5,32 & 5,22 & 5,44 & 4,62 & 4,60 & 4,60 & 4,63 & \\
\hline & 7.000 & 5,57 & 5,49 & 5,73 & 4,96 & 4,94 & 4,96 & 4,43 & \\
\hline \multirow{5}{*}{$6,75 \%$} & 4.000 & 4,60 & 4,47 & 4,62 & 3,59 & 3,56 & 3,55 & 3,56 & \\
\hline & 4.286 & 4,66 & 4,54 & 4,70 & 3,68 & 3,65 & 3,64 & 3,65 & \\
\hline & 5.000 & 4,83 & 4,71 & 4,88 & 3,89 & 3,87 & 3,86 & 3,88 & \\
\hline & 6.000 & 5,05 & 4,95 & 5,15 & 4,20 & 3,87 & 4,18 & 4,20 & \\
\hline & 7.000 & 5,28 & 5,19 & 5,41 & 4,50 & 4,17 & 4,49 & 4,20 & \\
\hline \multirow{5}{*}{$6 \%$} & 4.000 & 4,47 & 4,33 & 4,48 & 3,39 & 3,36 & 3,35 & 3,36 & \\
\hline & 4.286 & 4,53 & 4,40 & 4,55 & 3,47 & 3,44 & 3,43 & 3,44 & \\
\hline & 5.000 & 4,68 & 4,56 & 4,73 & 3,67 & 3,65 & 3,64 & 3,66 & \\
\hline & 6.000 & 4,89 & 4,78 & 4,97 & 3,96 & 3,93 & 3,93 & 3,95 & \\
\hline & 7.000 & 5,10 & 5,01 & 5,22 & 4,24 & 4,22 & 4,23 & 3,95 & \\
\hline \multirow{5}{*}{$4 \%$} & 4.000 & 4,11 & 3,98 & 4,10 & 2,90 & 2,88 & 2,86 & 2,86 & \\
\hline & 4.286 & 4,16 & 4,03 & 4,16 & 2,98 & 2,94 & 2,93 & 2,93 & \\
\hline & 5.000 & 4,29 & 4,17 & 4,31 & 3,13 & 3,11 & 3,10 & 3,10 & \\
\hline & 6.000 & 4,47 & 4,36 & 4,52 & 3,36 & 3,34 & 3,34 & 3,35 & \\
\hline & 7.000 & 4,65 & 4,55 & 4,73 & 3,59 & 3,57 & 3,57 & 3,59 & \\
\hline
\end{tabular}

Calculando a média dos resultados da análise de sensibilidade do CMPr, o eucalipto apresenta um CMPr da unidade energética em média de $\mathrm{R} \$ 4,92 / \mathrm{Gj}$ no caso do tratamento adensado e de $\mathrm{R} \$ 4,03 / \mathrm{Gj}$ no caso do tratamento tradicional, a média geral sendo de $\mathrm{R} \$ 4,41 / \mathrm{Gj}$, variando de $\mathrm{R} \$ 2,86 / \mathrm{Gj}$ a $\mathrm{R} \$ 6,26 / \mathrm{Gj}$. O coeficiente de variação do conjunto dos CMPr foi de $14,95 \%$ demonstrando uma boa homogeneidade dos dados em torno da média geral.
Pelo teste F, é possível afirmar que os dois tratamentos são estatisticamente diferentes ao nível de $1 \%$ de probabilidade.

\subsection{Custo de produção da unidade energética da biomassa de capim elefante}

A análise de sensibilidade foi realizada para duas opções de cultivos de capim-elefante o FBN e o capim

Cerne, Lavras, v. 17, n. 3, p. 417-426, jul./set. 2011 
híbrido Carajás. O primeiro, com um custo de adubação mais barato, mas, normalmente, com uma produção menor de biomassa.

A análise de sensibilidade (Tabela 8) permitiu mostrar os fatores que têm maior influência na formação do custo médio de produção da unidade energética $(\mathrm{R} \$ / \mathrm{Gj})$.
Mudando a taxa de juros, o custo da terra e a produção em cada cultivo, pode-se perceber a forte influência do volume de biomassa produzida na formação do CMPr. As oscilações do custo da terra e da taxa de juros não são preponderantes, como o é a quantidade de biomassa para atingir um menor CMPr.

Tabela 8 - Tabela comparativa do $\mathrm{CMPr}(\mathrm{R} \$ / \mathrm{Gj})$ do eucalipto com o do capim-elefante.

Table 8 - Comparative table of Eucalyptus and elephant grass Average Production Cost $(R \$ / G j)$.

\begin{tabular}{|c|c|c|c|c|c|c|c|c|c|c|c|}
\hline \multirow[b]{3}{*}{$\begin{array}{l}\text { Taxa de } \\
\text { juros } \\
\text { (\% a.a) }\end{array}$} & \multirow{2}{*}{\multicolumn{3}{|c|}{$\begin{array}{c}\text { Eucalipto } \\
\text { Produção média na } \\
\text { idade de colheita } \\
\left(\mathrm{m}^{3} / \text { ha.ano }\right)\end{array}$}} & \multicolumn{8}{|c|}{ Capim elefante } \\
\hline & & & & & $\begin{array}{r}\text { Variedade } \\
\mathrm{O} \mathrm{k} \\
\text { (t. MS }\end{array}$ & $\begin{array}{l}\text { om FBN. } \\
\mathrm{J} / \text { ha } \\
\text { a.ano) }\end{array}$ & & & $\begin{array}{r}\text { rido } \mathrm{C} \\
\mathrm{C} \\
\text { (t. } \mathrm{M}\end{array}$ & $\begin{array}{l}\text { m elefó } \\
\text { ás } \\
\text { a.ano) }\end{array}$ & \\
\hline & $\begin{array}{c}\text { Preço líquido } \\
\text { da terra } \\
(\mathrm{R} \$ / \mathrm{ha})\end{array}$ & 37,85 & 35,9 & 20 & 25 & 30 & 35 & 25 & 30 & 35 & 40 \\
\hline \multirow{5}{*}{$10 \%$} & 4.000 & 4,49 & 4,49 & 7,45 & 5,96 & 4,97 & 4,26 & 6,86 & 5,71 & 4,90 & 4,29 \\
\hline & 4.286 & 4,61 & 4,61 & 7,54 & 6,03 & 5,02 & 4,31 & 6,92 & 5,77 & 4,94 & 4,33 \\
\hline & 5.000 & 4,91 & 4,91 & 7,74 & 6,19 & 5,16 & 4,42 & 7,09 & 5,91 & 5,06 & 4,43 \\
\hline & 6.000 & 5,33 & 5,34 & 8,03 & 6,42 & 5,35 & 4,59 & 7,32 & 6,10 & 5,23 & 4,57 \\
\hline & 7.000 & 5,75 & 5,77 & 8,32 & 6,66 & 5,55 & 4,75 & 7,55 & 6,29 & 5,39 & 4,72 \\
\hline \multirow{5}{*}{$8 \%$} & 4.000 & 3,90 & 3,89 & 7,19 & 5,75 & 4,79 & 4,11 & 6,64 & 5,53 & 4,74 & 4,15 \\
\hline & 4.286 & 4,00 & 3,99 & 7,25 & 5,80 & 4,83 & 4,14 & 6,70 & 5,58 & 4,78 & 4,18 \\
\hline & 5.000 & 4,25 & 4,25 & 7,42 & 5,94 & 4,95 & 4,24 & 6,83 & 5,69 & 4,88 & 4,27 \\
\hline & 6.000 & 4,59 & 4,60 & 7,65 & 6,12 & 5,10 & 4,37 & 7,02 & 5,85 & 5,01 & 4,39 \\
\hline & 7.000 & 4,94 & 4,96 & 7,89 & 6,31 & 5,26 & 4,51 & 7,20 & 6,00 & 5,15 & 4,50 \\
\hline \multirow{5}{*}{$6,75 \%$} & 4.000 & 3,56 & 3,55 & 7,01 & 5,61 & 4,68 & 4,01 & 6,51 & 5,42 & 4,65 & 4,07 \\
\hline & 4.286 & 3,65 & 3,64 & 7,07 & 5,66 & 4,71 & 4,04 & 6,55 & 5,46 & 4,68 & 4,09 \\
\hline & 5.000 & 3,87 & 3,86 & 7,21 & 5,77 & 4,81 & 4,12 & 6,66 & 5,55 & 4,76 & 4,17 \\
\hline & 6.000 & 3,87 & 4,18 & 7,41 & 5,93 & 4,94 & 4,24 & 6,82 & 5,69 & 4,87 & 4,27 \\
\hline & 7.000 & 4,17 & 4,49 & 7,61 & 6,09 & 5,08 & 4,35 & 6,98 & 5,82 & 4,99 & 4,36 \\
\hline \multirow{5}{*}{$6 \%$} & 4.000 & 3,36 & 3,35 & 6,91 & 5,53 & 4,61 & 3,95 & 6,42 & 5,35 & 4,59 & 4,01 \\
\hline & 4.286 & 3,44 & 3,43 & 6,96 & 5,57 & 4,64 & 3,98 & 6,46 & 5,39 & 4,62 & 4,04 \\
\hline & 5.000 & 3,65 & 3,64 & 7,09 & 5,67 & 4,73 & 4,05 & 6,57 & 5,47 & 4,69 & 4,10 \\
\hline & 6.000 & 3,93 & 3,93 & 7,27 & 5,81 & 4,85 & 4,15 & 6,71 & 5,59 & 4,79 & 4,19 \\
\hline & 7.000 & 4,22 & 4,23 & 7,45 & 5,96 & 4,96 & 4,25 & 6,85 & 5,71 & 4,89 & 4,28 \\
\hline \multirow{5}{*}{$4 \%$} & 4.000 & 2,88 & 2,86 & 6,63 & 5,31 & 4,42 & 3,79 & 6,20 & 5,17 & 4,43 & 3,87 \\
\hline & 4.286 & 2,94 & 2,93 & 6,67 & 5,33 & 4,44 & 3,81 & 6,23 & 5,19 & 4,45 & 3,89 \\
\hline & 5.000 & 3,11 & 3,10 & 6,75 & 5,40 & 4,50 & 3,86 & 6,30 & 5,25 & 4,50 & 3,93 \\
\hline & 6.000 & 3,34 & 3,33 & 6,87 & 5,50 & 4,58 & 3,93 & 6,39 & 5,33 & 4,57 & 3,99 \\
\hline & 7.000 & 3,57 & 3,57 & 6,99 & 5,59 & 4,66 & 4,00 & 6,49 & 5,41 & 4,63 & 4,05 \\
\hline
\end{tabular}

Cerne, Lavras, v. 17, n. 3, p. 417-426, jul./set. 2011 
O CMPr do capim-elefante FBN foi estimado, em média, a $\mathrm{R} \$ 5,54 / \mathrm{Gj}$, enquanto o CMPr do capim-elefante Carajás atinge em média $\mathrm{R} \$ 5,34 / \mathrm{Gj}$. Em média, o CMPr da unidade energética do capim-elefante foi de $\mathrm{R} \$ 5,44 / \mathrm{Gj}$, variando de $\mathrm{R} \$ 3,79$ a $8,32 / \mathrm{Gj}$. O coeficiente de variação calculado foi de $20,44 \%$, sinal de uma boa homogeneidade dos dados. A análise de variância demonstrou com o teste $\mathrm{F}$ a $5 \%$ de probabilidade, que não há diferença estatisticamente significativa entre os dois cultivos do ponto de vista do custo médio de produção.

\subsection{Comparação capim elefante - eucalipto}

Na Tabela 8, apresentam-se os resultados da análise de sensibilidade do capim-elefante nas duas formas de cultivo e do eucalipto em seus dois melhores resultados no plantio convencional. Variaram-se os valores de taxa de juros e preço da terra para que, junto das produções, possam compor o melhor custo.

As análises de sensibilidade do custo médio de produção das duas culturas energéticas mostram que o eucalipto começa a ficar menos competitivo somente em certas condições: quando a elevada taxa de juros igual ou superior a $8 \%$ a.a. se associa a um elevado preço da terra e quando o capim-elefante atinge um patamar de produção igual ou superior a 35 toneladas/ha.ano.

A formação do CMPr do capim-elefante é fortemente influenciada pela quantidade de biomassa produzida, mais que por outros fatores como o preço da terra ou a taxa de juros. Entretanto, para o eucalipto, a taxa de juros tem maior influência na formação do CMPr.

A alta produção de matéria seca conseguida em plantios de capim-elefante não remunera suficientemente os elevados custos de seu manejo. Assim como a alta produção dos plantios adensados de eucalipto não remunera custos importantes como o de muda que ocorre 3 vezes, durante o ciclo de 18 anos.

Consequentemente, para projetos de implantação de termoelétricas no Brasil, seria possível usar cultivos de capim-elefante ou de eucalipto adensado nos primeiros anos para a geração da energia inicial. Com o tempo, os plantios convencionais chegarão à maturidade (por volta do sexto ano), tornando-se mais competitivos que as alternativas.

A análise de variância através do teste $F$ demonstrou que as quatro culturas (eucalipto adensado e convencional, capim-elefante FBN e híbrido Carajás) são, significativamente, diferentes entre si ao nível de $1 \%$ de probabilidade. O coeficiente de variação calculado foi de 18,59\% demonstrando uma boa consistência dos dados, ou seja, pouca dispersão do conjunto de dados em torno da média geral. Aplicando-se o teste de média de NewmanKeuls a $1 \%$ de probabilidade (Tabela 8), foi possível afirmar que os custos das duas cultivares de capim-elefante são iguais entre si e superiores aos do eucalipto. $\mathrm{O}$ teste de Newman-Keuls confirmou que as médias do CMPr dos dois tratamentos aplicados ao eucalipto são estatisticamente diferentes e que o tratamento convencional tornou-se preferível economicamente (Tabela 9).

Tabela 9 - Resultado do teste de Newman-Keuls ( $p<0,01)$ comparando as média do CMPr das culturas energética de capim elefante e de eucalipto.

Table 9-Result of the Newman-Keuls test ( $p<0,01$ ) comparating the averages of the Average Production Cost of the energetic crops of elephant grass and eucalyptus.

\begin{tabular}{lcccc}
\hline Cultura lignocelulósica para energia & Média & Comparações \\
\hline Capim-elefante FBN & 5,54 & A & \\
Capim hibrido Carajás & 5,34 & A & & \\
Eucalipto em plantio adensado & 4,92 & & B & \\
Eucalipto em plantio tradicional & 4,03 & & & C \\
\hline
\end{tabular}

\section{CONCLUSÕES}

O eucalipto apresenta um custo médio de produção do gigajoule inferior ao do capim-elefante.

O capim-elefante torna-se mais competitivo que o eucalipto em condições de elevados preços da terra, taxa de juros e produção de biomassa.

Para uma mesma taxa de juros, maiores custos da terra aumentam o custo médio de produção do gigajoule em todos os casos, e, para diferentes taxas de juros, o aumento do preço da terra causa aumento no custo médio de produção do gigajoule.

A taxa de juros é o fator que mais impacta o custo médio de produção da biomassa de eucalipto, enquanto que para o capim-elefante o maior impacto é da produtividade.

A biomassa para energia oriunda de plantios do eucalipto apresenta um custo médio de produção inferior aos preços de outras fontes de energia, tornando a atividade competitiva.

\section{AGRADECIMENTOS}

Os autores agradecem a RENABIO e a ArcelorMittal BioEnergia pela disponibilização dos dados sobre eucalipto.

Cerne, Lavras, v. 17, n. 3, p. 417-426, jul./set. 2011 


\section{REFERÊNCIAS}

BULLARD, M. Economics of Miscanthus production. In: JONES, M. B.; WALSH, M. (Ed.). Miscanthus: for energy and fibre. London: Science, 2001. p. 155-171.

COOMBS, J. Biomass energy: an industry waiting for growth. Renewable Energy, London, v. 5, n. 4, p. 133-145, 2002.

CRUZ, P. T. A.; NOGUEIRA, M. F. M. Oportunidades da biomassa energética no Brasil. Biomassa e Energia, Piracicaba, v. 1, n. 1, p. 37-44, 2004.

FLORES, R. A. Produção de capim elefante (Pennisetum purpureum Schum.) para fins energéticos no cerrado: resposta a adubação Nitrogenada e idade de corte. 2009. 66 p. Dissertação (Mestrado em Agronomia) - Universidade Federal Rural do Rio de Janeiro, Seropédica, 2009.

GOLDEMBERG, J. Biomassa e energia. Química Nova, São Paulo, v. 32, n. 3, p. 582-587, 2009.

LEITE, L. T. Produção de carvão vegetal. Curitiba: TECPAR, 2005. 6 p.

LOPES, B. A. O capim-elefante. Viçosa, MG: UFV, 2004. $56 \mathrm{p}$.

MAZARELLA, V.; URQUIAGA, S. Capim elefante como fonte de biomassa para a siderurgia. In: WORKSHOP SOBRE PRODUÇÃO SUSTENTÁVEL DE FERRO-GUSA, 2006, Ouro Preto. Anais... Ouro Preto: UFOP, 2006. Disponível em: <http://www.abmbrasil.com.br/cim/download/10h45\%20 Mazzarela.pps>. Acesso em: 25 nov. 2009.

PIMENTEL-GOMES, F.; GARCIA, C. H. Estatística aplicada a experimentos

agronômicos e florestais: exposição com exemplos e orientações para uso de aplicativos. Piracicaba: FEALQ, 2002. $309 \mathrm{p}$.

QUESADA, D. M.; BODDEY, R. M.; REIS, V. M.; URQUIAGA, S. Parâmetros qualitativos de genótipos

Cerne, Lavras, v. 17, n. 3, p. 417-426, jul./set. 2011 de capim-elefante (Pennisetum purpureum Schum.) estudados para a produção de energia através da biomassa. Seropédica: Embrapa, 2004. 4 p.

REZENDE, J. L. P.; OLIVEIRA, A. D. Análise econômica e social de projetos florestais. 2. ed. Viçosa, MG: UFV, 2008. $386 \mathrm{p}$.

SAMSON, R.; MANI, S.; BODDEY, R.; SOKHANSANJ, S.; QUESADA, D.; URQUIAGA, S.; REIS, V.; HOLEM, C. The potential of $\mathrm{C} 4$ perennial grasses for developing a global BIOHEAT industry. Critical Reviews in Plant Sciences, Chicago, v. 24, p. 461-495, 2005.

SENELWAA, K.; SIMS, R. E. H. Fuel characteristics of short rotation forest biomass. Biomass and Bioenergy, Amsterdam, v. 17, p. 127-140, 1999.

SILVA, M. L.; REZENDE, J. L. P.; LIMA, V. B. J.; CORDEIRO, S. A.; COELHO, L. M. J. Métodos de cálculo do custo da terra na atividade florestal. Cerne, Lavras, v. 14, n. 1, p. 75-81, 2008.

SMEETS, E. M. W.; FAAIJ, A. P. C. The impact of sustainability criteria on the costs and potentials of bioenergy production: applied for case studies in Brazil and Ukraine. Biomass and Bioenergy, Amsterdam, v. 27, p. 247-251, 2009.

SMEETS, E. M. W.; LEWANDOWSKI, I. M.; FAAIJ, A. P. C. The economical and environmental performance of Miscanthus and Switchgrass production and supply chains in a European setting. Renewable and Sustainable Energy Reviews, New York, v. 13, p. 1230-1245, 2009.

VALE, A. T.; BRASIL, M. A. M.; CARVALHO, C. M.; VEIGA, R. A. A. Produção de energia do fuste de Eucalyptus grandis hill ex-maiden e Acacia mangium willd em diferentes níveis de adubação. Cerne, Lavras, v. 6, n. 1, p. 83-88, 2000.

\section{VILELA, H. Capim-elefante Carajás (Ex. Paraíso)} (Pennisetum hybridum). Disponível em: $<$ http://www. agronomia.com.br/conteudo/artigos/artigos_capim_elefante paraiso.htm>. Acesso em: 21 nov. 2009. 